\title{
Evolution d'une barre sableuse sous un courant permanent
}

\author{
H. Michallet, B. Camenen, S. Bottero, \\ Ph. Larroudé et E. Barthélemy \\ Laboratoire des Ecoulements Géophysiques et Industriels (UJF-INPG-CNRS), \\ BP53, 38041 Grenoble Cedex 9, France. \\ Email: herve.michallet@hmg.inpg.fr Tel:0476825060 Fax:0476 82 5271
}

\section{Résumé}

Des expériences ont été réalisés pour évaluer la paramétrisation du transport sédimentaire dans un courant permanent. Une barre de sable est placée perpendiculairement au courant permanent d'intensité $U$. Différentes valeurs de $U$ ont été considérées afin d'observer différents régimes de transport pour différentes tailles de sédiment. Pour nos essais, il apparaît que le débit solide dépend fortement de la vitesse de l'écoulement et peu du diamètre du sédiment.

\begin{abstract}
Experiments have been performed to evaluate the parametrization of sediment transport in a permanent flow. A sand dune has been set perpendicularly to a flow of intensity $U$. Different values of $U$ have been considered in order to observe different transport regimes with different sediment sizes. In our experiments, the sediment discharge depends strongly on the flow rate but hardly on the sediment size.
\end{abstract}

\section{Introduction}

En zones côtières, le transport de sédiments est parfois dominé par les courants de marée au-dessus de barres sableuses de grandes dimensions (e.g. Hulscher et al., 1993). De nombreuses études ont porté sur la caractérisation des écoulements permanents sur des fonds sédimentaires présentant des structures périodiques de petites ou moyennes dimensions: rides et dunes (Engelund \& Fredsøe, 1982). En réponse à l'hydrodynamique, ces structures sédimentaires s'établissent et modifient fortement la rugosité de fond et donc l'écoulement (Raudkivi, 1963). Au-dessus de ces ondes sableuses, l'écoulement est caractérisé par un décollement au sommet de la forme sédimentaire. Ce décollement, qui s'apparente au décollement derrière une marche, nécessite une modélisation de la turbulence pour décrire correctement le transport du sédiment et donc la dynamique des structures (e.g. Mendoza \& Shen, 1990).

Le cas de la déformation et de la propagation d'une dune isolée a été en revanche très peu étudié. Ce cas s'avère pourtant très intéressant pour la calibration des modèles de transport sédimentaire (Camenen \& Larroudé, 2000). Tout en représentant une forme naturelle, il montre les limites des formules de transport. Nous nous sommes donc attachés à réaliser des expériences dans 


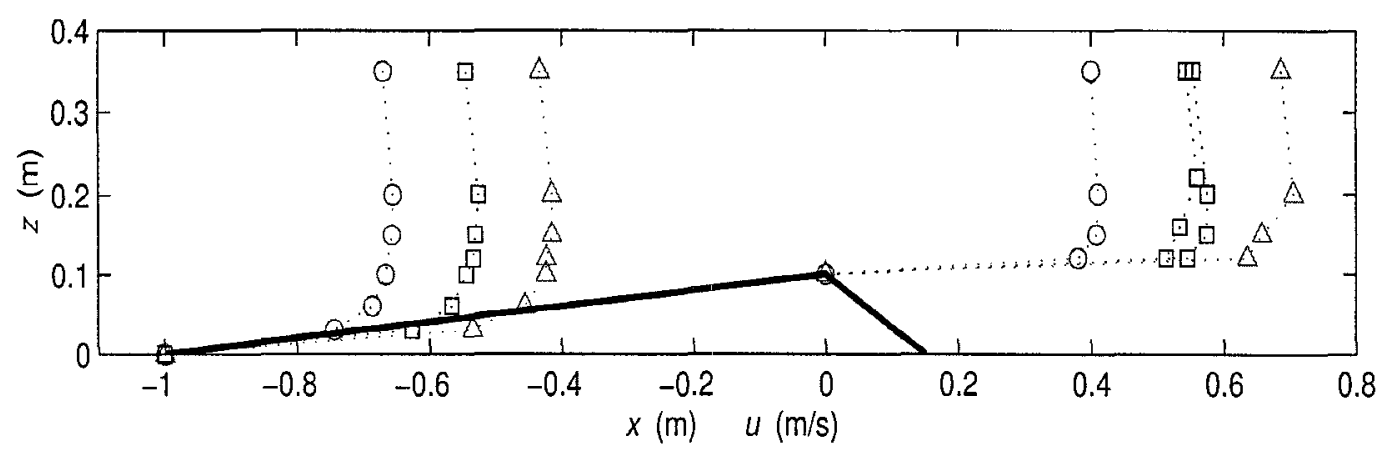

Figure 1: Profils de vitesse correspondant aux trois débits considérés, soient: $U=0.33,0.45$ et $0.56 \mathrm{~m} / \mathrm{s}$.

Figure 1: Velocity profiles related to the three flow rates considered: $U=0.33$, 0.45 and $0.56 \mathrm{~m} / \mathrm{s}$.

cette configuration afin d'évaluer les paramétrisations classiques du transport sédimentaire.

\section{Procédure expérimentale}

Dans un canal en hyppodrome permettant la circulation d'un courant uniforme (Piedra-Cueva et al., 1997), une dune de sable de hauteur $10 \mathrm{~cm}$ et de longueur $2 \mathrm{~m}$ est placée perpendiculairement au courant permanent d'intensité $U$. La profondeur d'eau est de $40 \mathrm{~cm}$. Nous avons considéré deux diamètres de grain $(d=0.2,0.9 \mathrm{~mm})$. Des essais ont été menés avec chaque taille, mais aussi avec un mélange des deux. L'évolution de la forme de la dune a été quantifiée par mesures video et par sondage acoustique. Une sonde à ultra-sons est ainsi placée au-dessus du lit sédimentaire et déplacée le long du canal à l'aide d'un charriot motorisé. Le temps de parcours de l'onde ultra-sonore est directement proportionnel à la distance séparant la sonde du fond sédimentaire.

Les profils de vitesse au début de la dune et à son sommet sont présentés Fig. 1 pour les trois débits considérés. Deux profils de vitesse sont présentés pour des conditions équivalentes $(U=0.45$ en $x=0)$ et donnent ainsi une idée des erreurs sur l'estimation des vitesses. La vitesse seuil de mise en mouvement des grains peut être estimée théoriquement sur fond plat d'après la courbe de Shields: on obtient $U_{c}=0.28$ et $0.39 \mathrm{~m} / \mathrm{s}$ pour $d=0.2$ et $0.9 \mathrm{~mm}$, respectivement. Expérimentalement, $U=0.33 \mathrm{~m} / \mathrm{s}$ correspond à peu près au début du transport au pied de la dune pour $d=0.9 \mathrm{~mm}$. Pour $U=0.56 \mathrm{~m} / \mathrm{s}$ et $d=0.2 \mathrm{~mm}$, un régime de sheet flow est observé où une certaine épaisseur du lit sédimentaire constituant la dune est en mouvement.

\section{Observations}

Deux déformations typiques sont représcntćes Fig. 2. Ces évolutions ont été déduites des mesures acoustiques. Dans le premier cas, la dune initiale est de forme symétrique. Au premier stade de la déformation, la dune s'asymétrise: 
(a)

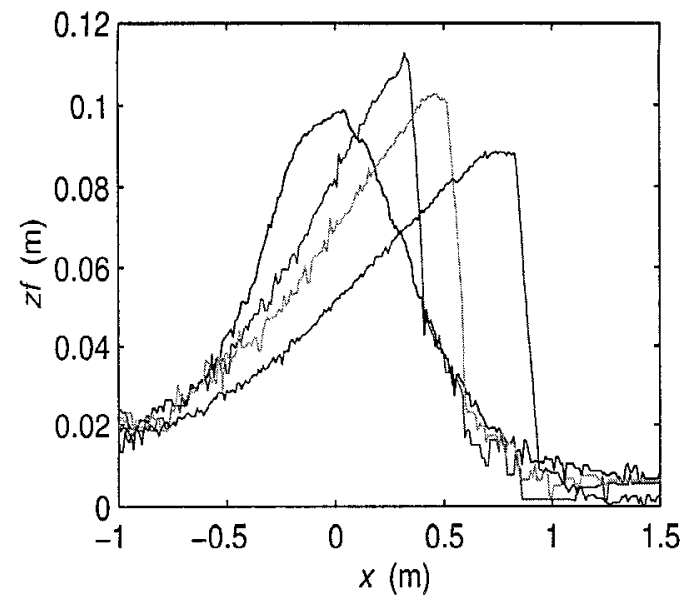

(b)

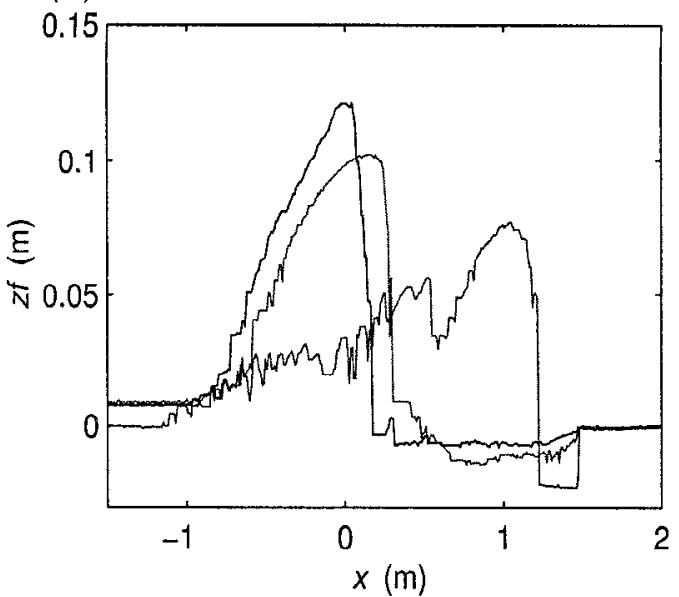

Figure 2: Profils expérimentaux de la dune: (a) à $t=0,10,27,50 \mathrm{mn}$ ( $U=0.45 \mathrm{~m} / \mathrm{s}, d=0.9 \mathrm{~mm}$ : régime de charriage); (b) à $t=0,6,47 \mathrm{mn}$ $(U=0.56 \mathrm{~m} / \mathrm{s}, d=0.2 \mathrm{~mm}$ : régime de sheet flow).

Figure 2: Experimental bar profiles: (a) at $t=0,10,27,50 \mathrm{mn}(U=$ $0.45 \mathrm{~m} / \mathrm{s}, d=0.9 \mathrm{~mm}$ : bed load regime); (b) at $t=0,6,47 \mathrm{mn}(U=0.56 \mathrm{~m} / \mathrm{s}$, $d=0.2 \mathrm{~mm}$ : sheet flow regime).

la pente amont devient plus douce tandis que la pente avale tend vers l'angle de frottement du sédiment au repos $\left(30^{\circ} \leq \phi \leq 35^{\circ}\right)$. Ceci s'explique du fait que l'écoulement décolle du fond sableux au sommet de la dune et les grains de sable chutent à peu près librement en aval. Pour cet exemple (Fig. 2a) l'écoulement décolle en fait un peu avant le sommet de la dune ce qui explique que la hauteur de la dune augmente dans les 10 premières minutes de la déformation. Dans un deuxième temps, la barre asymétrique se propage de manière quasi-stationaire, en perdant de la hauteur.

Pour le deuxième exemple (Fig. 2b), la forme initiale a été choisie proche du profil quasi-stationaire. Pour cet essai (sédiment fin et vitesse d'écoulement importante), le régime est de sheet flow. L'évolution de la dune est similaire à l'essai représenté Fig. 2a qui correspondait à un régime de charriage. Nous notons toutefois des irrégularités sur la pente amont de la dune correspondant à la formation de rides. Dans le régime intermédiaire $(U=0.45 \mathrm{~m} / \mathrm{s}$ et $d=0.2 \mathrm{~mm})$ ces rides s'amplifient très rapidement, d'importantes structures turbulentes se développent au fond et les structures sédimentaires deviennent pleinement tridimensionnelles.

Pour chacun des deux essais présentés Fig. 2, la surface sous les profils est conservée à, respectivement, $7 \%$ et $4 \%$ près. Aucune tendance n'est décelée, ces écarts semblent provenir de l'erreur de mesure, en particulier liée aux irrégularités transverses (la progression de la dune n'est pas parfaitement bidimensionnelle). Nous considérons donc que nous vérifions la conservation de la masse et que la quantité de sédiment en suspension est négligeable devant 
la quantité de sédiment transportée par charriage.

Nous représentons Fig. 3 les évolutions de la hauteur de dune et de la vitesse de son sommet pour les différents essais réalisés. Ces évolutions paraissent assez irrégulières. En effet, alternativement, le sédiment s'accumule au sommet de la dune et s'écoule par avalanche sur la pente avale. Le processus d'évolution n'est donc pas temporellement uniforme. De plus, ce processus n'est pas uniforme transversalement. Ceci induit des erreurs dans l'estimation de l'évolution moyenne de la dune. En particulier, Fig. 3b, deux estimations de la vitesse d'avance de la dune sont représentées pour $U=0.45 \mathrm{~m} / \mathrm{s}$ et $d=0.9 \mathrm{~mm}$ : l'une d'après le sondage acoustique; l'autre d'après le suivi video sur la paroi du canal. Ces deux estimations sont assez proches et donnent une idée des effets transverses.

Il apparaît que la décroissance de la hauteur de la dune est plus forte pour les essais réalisés avec le sédiment fin (Fig. 1a). Par ailleurs, il apparaît clairement Fig. 3b que la vitesse d'avance de la dune dépend fortement de la vitesse de l'écoulement mais très peu du diamètre du sédiment.

Si nous négligeons le transport solide par suspension, le débit solide au sommet (par unité de largeur) peut être estimé simplement par le dépôt sédimentaire à l'aval de la dune:

$$
q_{s}=V_{d}(1-n) H
$$

où $n=0.35$ est la porosité estimé du lit déposé. L'évolution du débit solide au cours du temps pour nos différents essais est reportée Fig. 3c. Le débit solide augmente avec la vitesse de l'écoulement. Pour une même vitesse, le sable fin et le sable mixte induisent un débit solide sensiblement identique et légèrement plus faible que pour le sédiment de plus gros diamètre.

\section{Modélisation}

L'équation de conservation de la masse s'écrit (Fredsøe \& Deigaard, 1992) :

$$
\frac{\partial z_{f}}{\partial t}+\frac{\partial q_{s}}{\partial x}=0
$$

où $z_{f}$ est la cote du fond. Elle est résolue numériquement par méthode des volumes finis pour prédire la déformation et l'évolution de la barre. Le transport solide pour le modèle est défini tel que:

$$
q_{s}=A U^{n}\left(1-\beta \frac{\mathrm{d} z_{f}}{\mathrm{~d} x}\right)
$$

Cette formulation est employée par Bailard (1981), où le coefficient $n$ est pris égal à 3 si l'on suppose un transport solide par charriage. Le coefficient $A$ est uniquement fonction du frottement au fond, donc du diamètre du sédiment $d$ si l'on considère une rugosité de peau sur fond plat: $A=4.710^{-5} \mathrm{~s}^{2}$ pour $d=0.2 \mathrm{~mm}$ et $A=6.810^{-5} \mathrm{~s}^{2}$ pour $d=0.9 \mathrm{~mm}$. Ces valeurs vérifient que le débit solide est légèrement plus fort pour le grain le plus gros. Le coefficient $\beta$ est souvent introduit pour prendre en compte l'effet de la pente du fond sur 
(a)

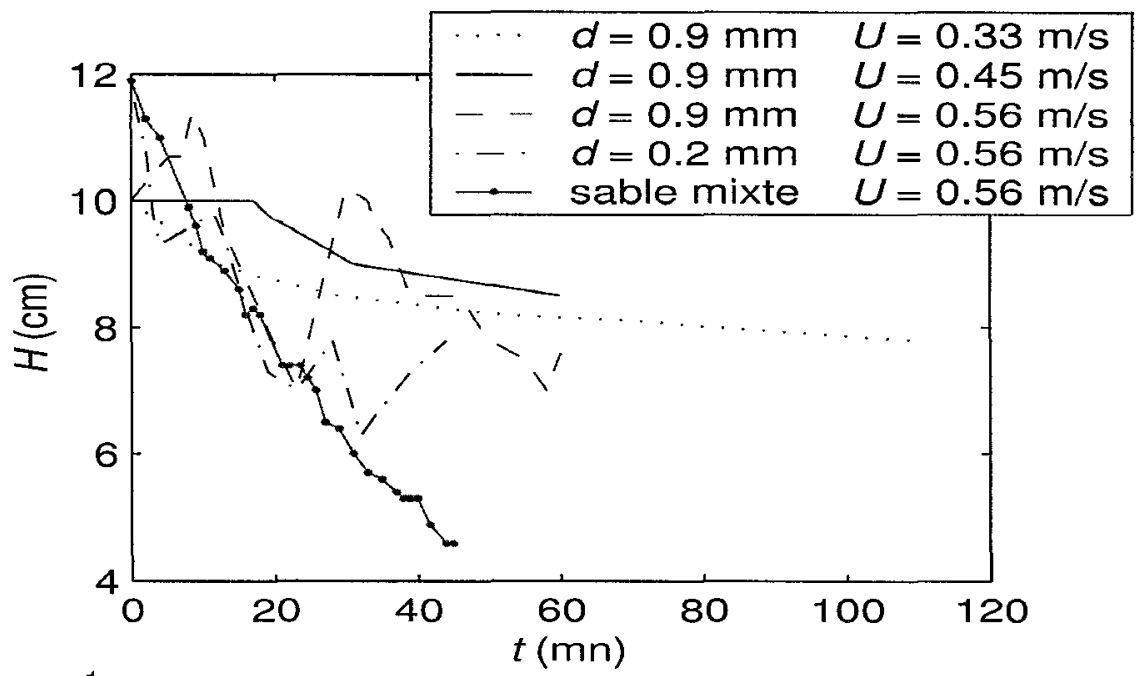

(b)

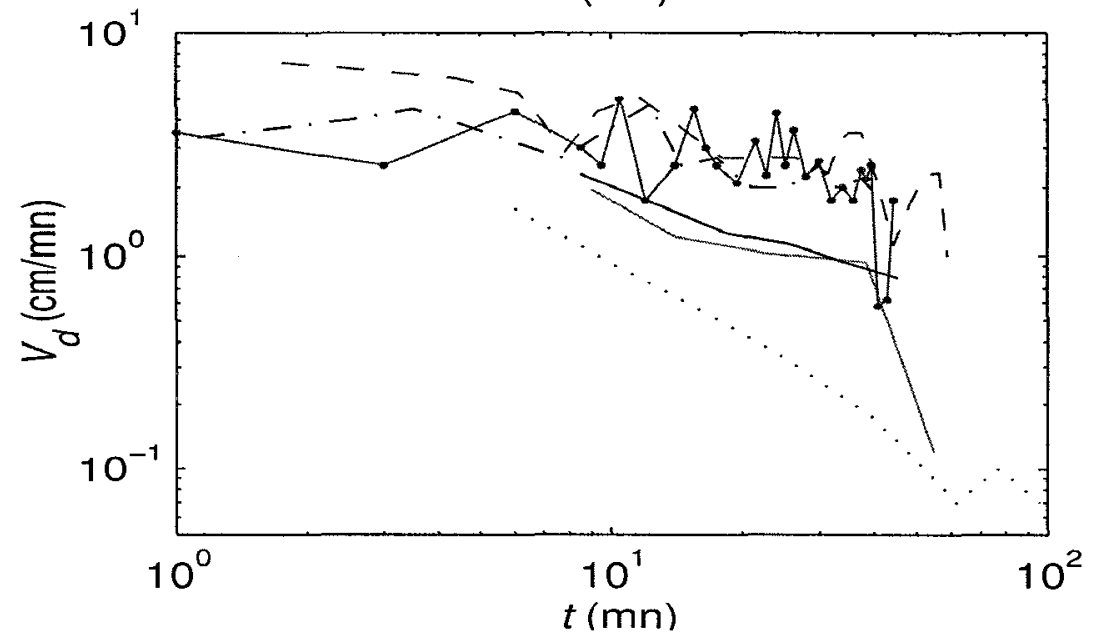

(c)

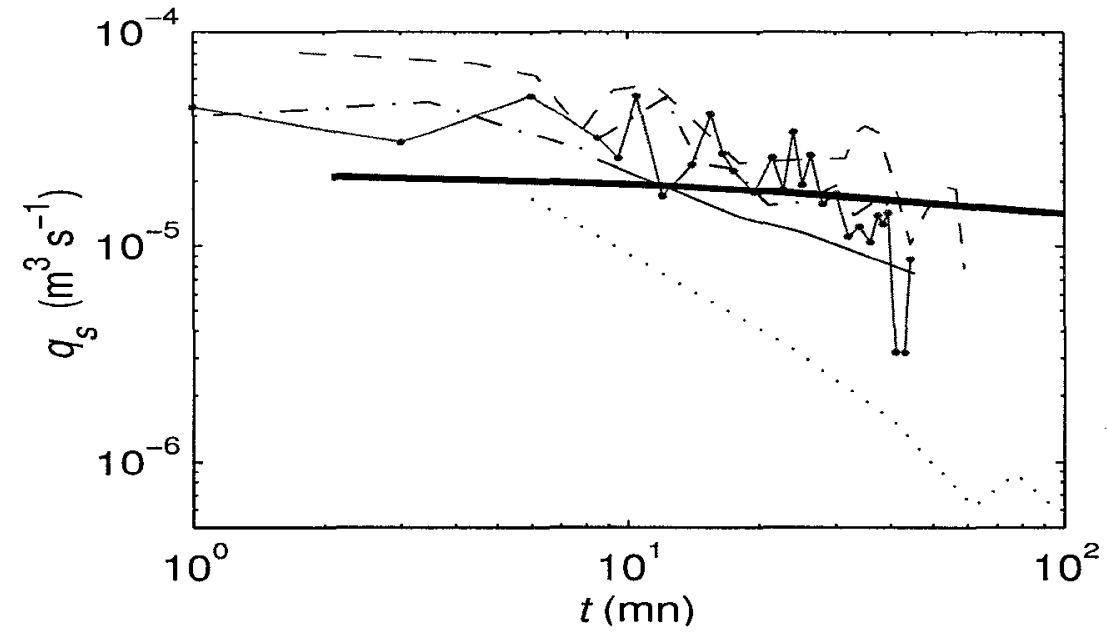

Figure 3: Evolution au cours du temps (a) de la hauteur de la dune, (b) de la vitesse du sommet et (c) du débit solide au sommet (1), où la courbe en trait gras est obtenu numériquement (cas de la Fig. 4b).

Figure 3: Evolution against time of (a) the dune height, (b) sand bar velocity and (c) sediment discharge at the top of the dune (1), where the thick solid line is obtained numerically (for $U=0.45 \mathrm{~m} / \mathrm{s}, A=6.810^{-5} \mathrm{~s}^{2}$ and $\beta=1.7$ ). 
le transport sédimentaire (débit solide réduit ou facilité par gravité), tel que $\beta=(\tan \phi)^{-1}$.

Des profils de la déformation de la dune sont présentés Fig. 4 pour deux valeurs du coefficient $\beta$. Il est important de souligner que le modèle ne prend pas en compte le décollement de l'écoulement au sommet de la dune. Pour $\beta=0$, le transport calculé est donc identique de part et d'autre de la dune et augmente avec $z$. Ceci implique le raidissement de la dune, sans perte de hauteur (Fig. 4a). Lorsque la pente avale devient trop forte, le calcul numérique diverge au sommet. Il est toutefois remarquable que le modèle numérique parvient à propager le choc qui résulte du raidissement de la dune. Le choix d'une valeur non nulle de $\beta$ permet d'éviter la divergence du calcul au niveau du sommet de la dune (Fig. 4b). Les autres grandeurs $\left(V_{d}, q_{s}\right)$ dépendent très peu de la valeur de $\beta$.

Il apparaît ainsi que les vitesses de déplacement de dune estimées par le modèle sont 3 à 4 fois supérieures à celles mesurées expérimentalement (la dune avance d'environ $50 \mathrm{~cm}$ en $40 \mathrm{mn}$ pour l'essai de la Fig. 2a; et de $1.78 \mathrm{~m}$ en $45 \mathrm{mn}$ pour le calcul de la Fig. 4b).

Pour ce qui est du débit solide au sommet, les variations au cours du temps correspondant au calcul de la Fig. 4b sont comparées aux valeurs expérimentales sur la Fig. 3c. La courbe numérique (en trait gras) est donc à comparer avec la courbe en trait fin. On observe que le débit solide est plus important expérimentalement au départ, ce qui peut conduire à discuter du choix de la valeur de $A$ dans le modèle. La rugosité équivalente du fond sédimentaire avec la dune est ainsi sans doute supérieure à la rugosité de peau. Pour les temps plus grands, $q_{s}$ décroît plus fortement expérimentalement. Ceci s'explique en partie par une diminution de la rugosité équivalente. Par ailleurs, à mesure que la hauteur de dune diminue, la vitesse de l'écoulement au-dessus de la dune diminue également et se rapproche donc de la vitesse seuil de mise en mouvement des grains. Ceci a pour conséquence d'induire une décroissance forte du débit solide, d'autant plus forte que $U$ est proche de $U_{c}$. En particulier pour le cas $U=0.33 \mathrm{~m} / \mathrm{s}$, une modélisation plus judicieuse serait donc de considérer une équation du type:

$$
q_{s}=A\left(U^{2}-U_{c}^{2}\right)^{3 / 2}
$$

\section{Conclusion}

Nous avons considéré la propagation d'une barre sableuse de grande dimension relative dans un courant permanent. Nous avons observé que l'essentiel du transport sédimentaire s'effectue par charriage. La dynamique de la dune présente les mêmes caractéristiques dans la plupart des cas: la pente amont et la hauteur de la dune décroissent tandis que la pente avale est la pente de stabilité du sable au repos. Seul dans le cas d'un sédiment fin et d'une vitesse d'écoulement intermédiaire ( $U \simeq 0.45 \mathrm{~m} / \mathrm{s}$ ), des instabilités tridimensionnelles apparaissent sur le fond.

Ces expériences nous ont permis de discuter les principaux paramètres des 
(a)

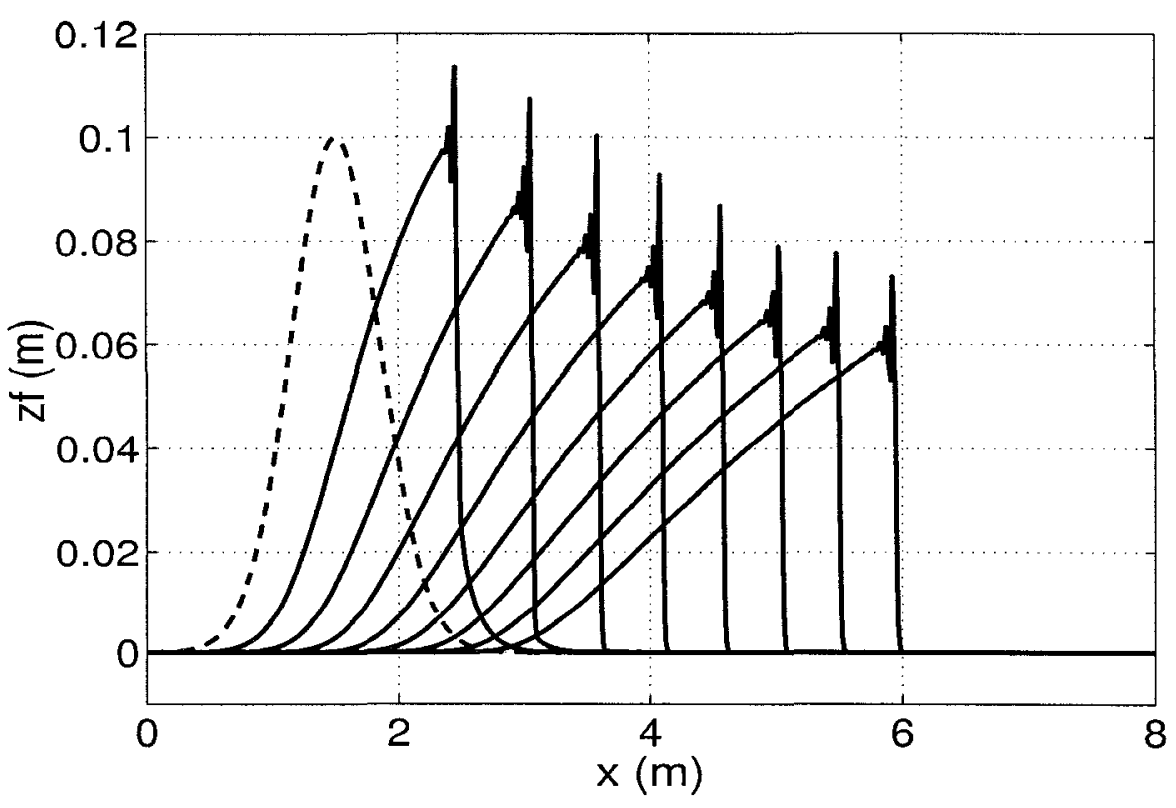

(b)

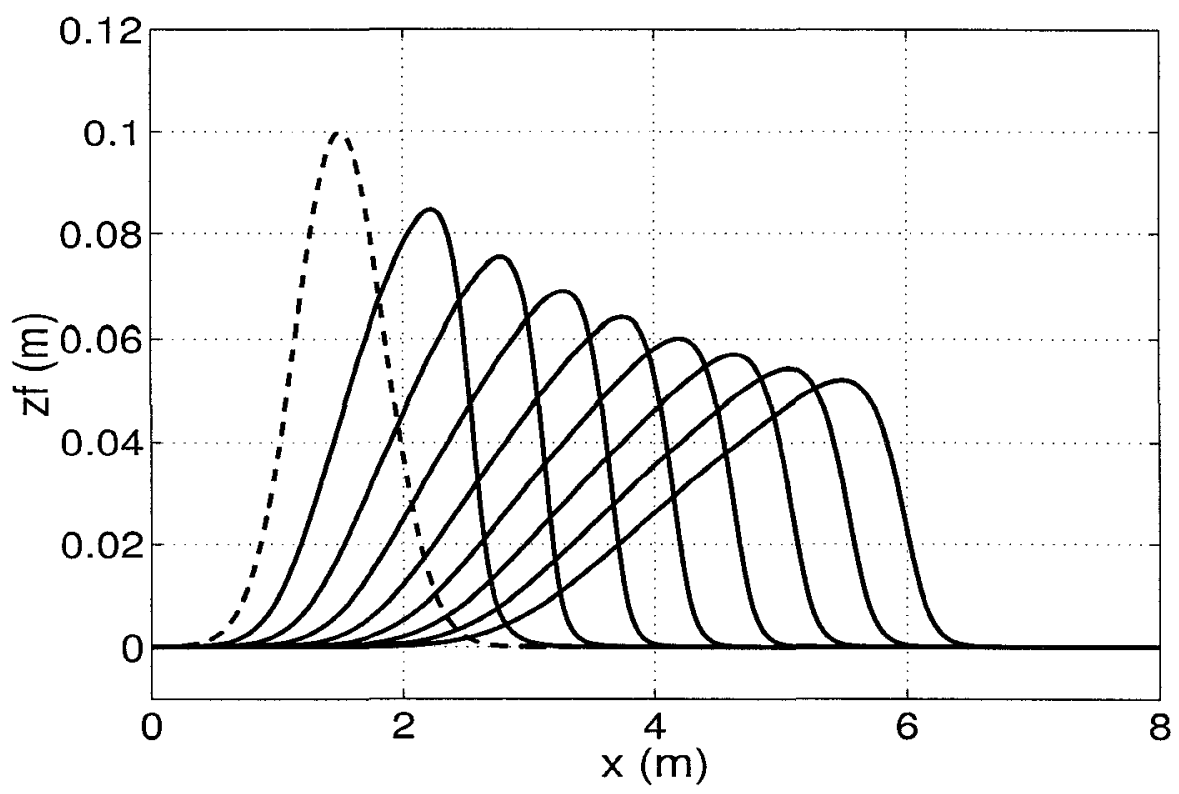

Figure 4: Profils numériques de la dune toutes les $15 \mathrm{mn}$ pour $\beta=0$ (a) et $\beta=1.7$ (b). ( $U=0.45 \mathrm{~m} / \mathrm{s}, A=6.810^{-5} \mathrm{~s}^{2}$ : cas de la Fig. 2a).

Figure 4: Numerical profiles of the dune every $15 \mathrm{mn}$ for $\beta=0$ (a) and $\beta=1.7$ (b). ( $U=0.45 \mathrm{~m} / \mathrm{s}, A=6.810^{-5} \mathrm{~s}^{2}$ : case of Fig. 2a). 
formules de transport. Il apparait en particulier que l'effet de pente, initialement introduit pour faciliter (réduire) le transport sur des pentes descendantes (ascendantes), permet essentiellement de stabiliser le calcul numérique.

Des expériences sont actuellement en cours pour visualiser et ainsi mieux comprendre l'interaction de la turbulence avec le sédiment, en particulier au point de recollement de l'écoulement à l'aval de la dune. Nous projetons d'effectuer le même type d'expériences en ajoutant l'action d'une houle afin d'étudier les conditions de stabilité des formes sédimentaires sous l'influence d'une houle et d'un courant.

\section{Remerciements}

Ce travail a été réalisé en partie à l'aide d'un financement CNRS-SPI. Les expériences ont bénéficiées du support technique de J.-M. Barnoud, les commentaires pertinents de A. Temperville, L. Rossi, M. Kamalinezhad et N. Taylor ont été également fortement appréciés.

\section{Références}

BaIlard, J. A. 1981. An energetic total load sediment transport model for a plane sloping beach. J. Geophys. Res., 86(C11), 10938-10954.

Camenen, B., \& Larroudé, Ph. 2000. Numerical comparison of sediment transport formulae. Pages 37-42 of: Marine Sandwave Dynamics.

Engelund, F., \& Fredsøe, J. 1982. Sediment ripples and dunes. Annu. Rev. Fluid Mech., 14, 13-37.

Fredsøe, J., \& DeigaARd, R. 1992. Mechanics of coastal sediment transport. World Scientific. (pages 265-275).

Hulscher, S. J. M. H., De Swart, H. E., \& DE VRIend, H. J. 1993. The generation of offshore tidal sand banks and sand waves. Cont. Shelf Res., 1183-1204.

Mendoza, C., \& Shen, H. W. 1990. Investigation of turbulent flow over dunes. J. Hydr. Engrg., ASCE, 116(4), 459-477.

Piedra-Cueva, I., Mory, M., \& Temperville, A. 1997. A race-track recirculating flume for cohesive sediment research. IAHR J. Hydraul. Res., 35(3), $377-396$.

RaUdKIVI, A. J. 1963. Study of sediment ripple formation. J. Hyd. Div., ASCE, 89(HY6), 15-33. 